\title{
ONCOGENIC LIVER FLUKES ACQUIRED FROM EATING RAW OR UNDERCOOKED FISH AND CRABS
}

\author{
By \\ WAEL MORSY A. EL-SAYED ${ }^{1}$ ASHRAF A. ABOU-BAKR ${ }^{1}$ \\ and TOSSON A. MORSY ${ }^{2}$
}

Military Medical Academy, Cairo $11291^{1}$ and Faculty of Medicine, Ain Shams University, Cairo $11566^{2}$, Egypt

\section{Abstract}

Liver flukes as Clonorchis sinensis and the similar genus Opisthorchus felineus and O. viverrini are considered oncogenic as causing cholangiocarcinoma in about ten percent of cases where millions are infected in southeast Asia and Europe being endemic there and mostly asymptomatic but require treatment. Klatskin tumour is bile duct bifucational area carcinoma affection that is almost unique for these infections which comes by eating raw or undercooked fish and crabs. There has been strong evidence that both types of liver flukes are present in Egypt and Middle East being endemic since the snail first intermediate hosts is so many fresh water snails and risks are also, still similarly, to millions of humans, who require treatment to halt progression of disease in asymptomatic cases. This review aimed to clarify those types of flukes for health care workers and the public for better diagnosis, prevention and control, Also, to describe the main traits of $C$. sinensis and $O$. viverrini, their molecular biology and core carcinogenic mechanisms in a contrast pattern.

Keywords: Cholangiocarcinoma, Liver flukes, Fish, Crabs

\section{Introduction}

Clonorchis sinensis also known as the Chinese liver fluke is endemic in China, Japan, Taiwan, Vietnam, and Korea as well as in far eastern Russia. Dogs and cats are the common-est reservoirs (Qian et al, 2016). It is estimated that more than 35 million people are infected worldwide, with 600 million at risk. Opisthorchiasis is generally caused by the closely related species with similar eggs and clinical picture and are called: $O$. felineus or $O$. viverrini. These are liver flukes of cats, dogs, and other fish-eating mammals, which serve as reservoir hosts. $O$. felineus occurs in Southeast Asia and in Central and Eastern Europe; Germany, Italy, France and other parts Russia. Prevalence rates are 40 to $95 \%$ in some areas, and it is estimated that more than 16 million people are infected (WHO, 1995). O. viverrini is endemic in Thailand, Vietnam and Cambodia, and Laos and is estimated that worldwide over 23 million people are infected, including 15 million in China (Upatham et $a l, 1984)$. Adult flukes can remain in the bile ducts for 26 years and protective immunity does not develop and repeat infection (Tan et al, 2009).
Morsy and El Maridi (1978) in Jordan detected a case of clonorchiasis among the Refugees in Baqaa Camp. Al-Karawi and Qat$\tan$ (1992) in Saudi Arabia reported a case of C. sinensis and Al Karawi et al. (1993) reported clonorchiasis in 35 patients with the acute gallstone pancreatitis and/or cholangitis whom underwent endoscopic sphincterotomy. Stauffer et al. (2004) in USA reported infections in migrants from Laos, Cambodia and Thailand, the former Soviet Union and South America only clinical clue to infection was a mild absolute eosinophilia (500 to 1000 microL). Morsy and Al-Mathal (2011) in Egypt reported Clonorchis sinensis in Egyptian employees returning back from Saudi Arabia. Pozio et al. (2013) stated that in the $20^{\text {th }}$ Century, the majority of infections in humans have been reported in Eastern Europe (e.g., Belarus, Russia, and Ukraine) and Asia (Siberia). In EU in the last fifty years, the parasite has been detected in humans of Germany and Greece, and in the red foxes, polecats, cats, dogs, fish and mollusks of Germany, Italy, Poland, Portugal and Spain (Whitton, 1982). In Italy, four individual cases and eight outbreaks of opisthorchiasis were reported from 2003 to 
2011, for a total of 211 confirmed human cases. All patients had consumed raw fillets of tench or doctor fish (Tinca tinca) is a fresh- and brackish-water fish (family Cyprinid) found in two lakes in central Italy but some of infected people were tourists who developed the disease in their respective home-countries. Zheng et al. (2017) reported that $O$. viverrini and $C$. sinensis, are typical representatives causing cholangiocarcinoma (CCA), the second most common primary hepatic malignancy with dismal prognosis. O. viverrini is prevalent in Southeast Asia, infecting 10 million people while C. sinensis has a wider distribution, affecting more than 35 million people's health. They added that both have some common characteristics and/or discrepancies in life cycle, genome, and transcriptome.

In endemic areas, cholangiocarcinoma incidence was very high (Sithithaworn et al, 1984), but it is only about $10 \%$ of whole cases who are mostly asymptomatic. Dried or pickled fish can be a source of infection. Infected water by itself is not infectious but the infective meta-cercaria in fish meat or gills are infectious (Keiser and Utzinger, 2005) or even in imported raw fish (Yossepowitch et al, 2004) and in frozen or salted freshwater fish (Fan , 1998).

Life cycle: The life cycles of Clonorchis and Opisthorchis flukes are similar; they begin with release of embryonated eggs into the biliary ducts and subsequently the stool. Eggs are ingested by a snail (first intermediate host), where miracidia are released and go through several developmental stages (sporocysts, rediae, and cercariae). The cercariae are released from the snail into fresh water and subsequently penetrate the flesh of freshwater fish (second intermediate host), where they encyst as metacercariae. Animals (definitive hosts) or humans (incidental hosts) acquire infection via ingestion of raw, undercooked, salted, pickled, or smoked freshwater fish (Harinasuta and Harinasuta, 1984). The metacercariae excyst in the duodenum and ascend the biliary tract. Maturation to adult flukes takes approximately one month. The adults generally reside in small and medium sized biliary ducts; occasionally they reside in the gallbladder or pancreatic duct (Rim, 2005). The adults can remain in the bile ducts for up to 26 years (Wang et al, 2004). Freshwater snail, Parafossarulus manchouricus often that serves as a first intermediate host for $C$. sinensis in China, Japan, Korea, and Russia (Hung et al, 2015). Other snails include Alocinma longicornis, Bithynia longicornis, B. fuchsiana, B. misella, Parafossarulus anomalospiralis, Melanoides tuberculta, Semisulcospira cancellata, Koreoleptoxis anurensis, Semisulcospira libertina, and Assiminea lutea in China; and Melanoides tuberculata in Vietnam (Pinto et al, 2018). Opisthorchis first intermediate hosts are freshwater snails of the genus Bithynia, the only idenotified host was Bithynia siamensis with its three subspecies (Saijuntha et al, 2014).

In Egypt, at least 37 snail species of medical and veterinary importance belong to $11 \mathrm{fa}$ milies; Neritidae, Viviparidae, Valvatidae, Bithyniidae, Melaniidae, Hydrobiidae, Lymnaeidae, Succineidae, Planorbidae, Physidae and Ancylidae (Ibrahim et al, 1999). At the same time, Egypt depends on the Nile River as its main and almost exclusive source of fresh water as one of the African countries that could be vulnerable to water stress under climate change. Because of being at the bottom end of the river, it is affected by the climate change impacts, and invasion more fresh water snails. As to the second intermediate hosts of $C$. sinensis, Cyprinidae family of freshwater fish is the largest and is comprised of the carps and minnows serve as. They are toothless; primarily eat gastropods and aquatic vegetation, though some will eat algae, small fish, or other molluscs. Given their great diversity, wide geographic distribution (extensive in Europe, Asia, Africa, and North America), and preferred food, and given that these fish are one of the most important food fish for humans in much of the worldwide. In China, 102 species of fish and 
four species of shrimp are known to be the intermediate hosts (Chai et al, 2005). In Korea, 40 species of freshwater fish were recorded as hosts and seven species in Russia (Fattakhov et al, 2012). Dao et al. (2017) reported that a total of 12,000 snails belonging to six families, of which 1616 Bithynia snails representing $B$. siamensis goniomphalos and B. funiculata; as well as 754 fish representing 12 species were examined. Shedding of $O$. viverrini cercariae was observed only in B. s. goniomphalos and B. funicula$t a$, at infection rates of $0.86 \%$ and $0.14 \%$, respectively. $O$. viverrini metacercariae were found in ten fish species representing both Cyprinidae and non-Cyprinidae families. They added that Carassius auratus, a fish species commonly eaten raw, Rasbora aurotaenia and Puntius brevis had the highest prevalence of up to $74.0 \%$. Sharing of the same snail and fish intermediate host species was found for $O$. viverrini and the $\mathrm{O}$. viverrini duck-genotype.

Cats and dogs are actually known reservoir hosts for Opisthorchis, infected via consuming raw or undercooked contaminated fish, fish dishes or food remains from households, and they sustain parasite egg spread via open environment defecation (Tangkawattana and Tangkawattana, 2018). Usually the infected animals do not exhibit apparent any clinical symptoms or even any specific abnormalities in laboratory examinations. Nguyen et al. (2018) suggested that cats are transported over great distances, and this poses a risk of spreading liver fluckes.

Clinical picture: The clinical manifestations of Opisthorchis and Clonorchis are similar and may be considered together. Most infected individuals are asymptomatic and have a benign course. The risk of symptomatic infection and complications rises as the intensity and duration of infection increase. Light infections ( $<100$ flukes or up to 10,000 eggs/gram of stool) rarely cause symptoms. Even in heavily infected patients, symptoms occur in only about $10 \%$ of them (Pakharukova and, Mordvinov, 2016). Peri pheral eosinophilia may be present (usually up to 10 to $20 \%$ ), and serum IgE levels may be elevated.

Acute symptoms: Pathologic and clinical consequences of infection are related to the intensity and duration of cumulative infection. With acute $C$. sinensis and $O$. viverrini infection, most infected patients have low fluke counts and therefore are asymptomatic. Relatively heavy infection occurs in 5 to $10 \%$ of patients; these individuals have nonspecific symptoms, such as the right upper quadrant abdominal pain, indigestion, diarrhea, flatulence, and fatigue. With O. felineus infection, acute symptoms are more common, usually beginning 10 to 26 days after eating heavily infected undercooked fish, and manifesting as high-grade fever, anorexia, nausea, vomiting, abdominal pain, malaise, myalgia, arthralgia, malaise, and urticaria. Lymphadenopathy and tender hepatomegaly may occur. When symptoms occur, they usually last for two to four weeks. High levels of circulating eosinophils are common. Eggs generally become detectable in the stool after three to four weeks. Some variations in clinical presentations of clonorchiasis and opisthorchiasis may occur in different geographic areas. The O. felineus infection may be associated with the acute, serum sickness-like presentation (Lawley et al, 1984); C. sinensis infection may be associated with intrahepatic pigment stones as part of the calculus of bile duct, located proximal to the confluence of the left and/or right hepatic ducts The morbidity was up to $18.7 \%$ in Taiwan while the average morbidity was only $0.6 \%-1.3 \%$ in western countries (Tsui et al, 2011). Apart from bacteria, and both $C$. sinensis and $O$. viverrini, other parasites as Ascaris lumbricoides, and Schistosoma species account for intrahepatic stone as well not only damage the bile duct epithelium to cause inflammation but also form the nucleus of stones with their body (Ran et al, 2017).

Chronic symptoms and complications: Symptoms can occur late in the course of 
infection due to chronic mechanical injury and physical bile duct obstruction by the adult flukes, particularly in individuals with a heavy burden of adult worms. Symptoms can include fatigue, abdominal discomfort, anorexia, weight loss, dyspepsia, and diarrhea (Choi et al, 2003). An elevated alkaline phosphatase level may be observed, but transaminases are generally normal. The gallbladder is often nonfunctional and enlarged. Dead parasites or ova serve can serve as a nidus for stone formation. Severe disease can result in obstructive jaundice, pancreatitis, recurrent cholangitis, and pyogenic liver abscesses. More serious complications of chronic infection include the cholangitis, cholangiohepatitis, and cholangio-carcinoma (Mairiang et al, 2012). These complications result from chronic irritation to the epithelial cells, which desquamate and proliferate, eventually leading to the hyperplasia, dysplasia, and fibrosis. Periductal eosinophilic and lymphocytic infiltration of portal areas also occurs, suggesting a role for immunemediated tissue damage (Geller, 2014). These changes result in formation of pigment stones, biliary strictures, dilation of intrahepatic bile ducts, and fibrosis of hepatic cells. Such complications generally develop after the third decade of life. Patients with heavy Opisthorchis or Clonorchis infections may have a 15-fold higher risk of developing cholangiocarcinoma (Aksorn et al, 2018). The symptoms include jaundice, weight loss, epigastric pain, an abdominal mass, and ascites. Carcinogenesis mechanisms are unclear but may include chronic irritation, nitric oxide formation, intrinsic nitrosation, and activation of drug-metabolizing enzymes.

Diagnosis: Can be established by identifying eggs in stool, duodenal aspirates or bile specimens. Serology has been developed but is not widely available; imaging can be a useful adjunctive diagnostic tool. Diagnosis of clonorchiasis or opisthorchiasis should prompt evaluation of family members with stool microscopy and blood count for eosinophilia. In the setting of eosinophilia with negative stool microscopy, ultrasound may be useful. Eggs may be visible in the stool approximately four weeks following infection. Eggs may not be present in light infections or may be detectable only in concentrated specimens. It is difficult to distinguish between Clonorchis and Opisthorchis eggs; both are oval in shape and measure approximately 20 to 30 microns by 15 microns (Choi et al, 2005). But, there were still difficulties in distinguishing them from eggs of heterophyid and lecithodendriid parasites (Kaewkes et al, 1991). Adult worms may be seen at endoscopy or endoscopic retrograde cholangiopancreatography (ERCP). They may also be passed in the stool following anthelminthic therapy. The adult flukes range in size from a few millimeters to several centimeters. Adult $C$. sinensis worms are flat and elongated, measuring 10 to $25 \times 35 \mathrm{~mm}$ wide. Adult $O$. felineus and $O$. viverrini are usually 8 to $12 \mathrm{~mm}$ long, but $O$. felineus is wider ( 2 to $3 \mathrm{~mm}$ compared with 1 to $2 \mathrm{~mm}$ ).

Laboratory assays: Serologic testing for opisthorchiasis and clonorchiasis by ELISA and radioimmunoprecipitation and immunoblotting (a significant cross-reactivity) were used in some laboratories but were not widely available (Sirisinha et $\mathrm{al}, 1990)$. The assay cannot reliably distinguish between current and past infection and also may crossreact with other parasites. Development of more sensitive and specific assays is under investigation, but, thus far, these are not widely available. One monoclonal antibody based dot-ELISA has been developed using purified $O$. viverrini antigen; excellent sensitivity and specificity have been observed. Immunoblot assays using $C$. sinensis-specific excretory-secretory antigens have been developed with variable sensitivity (76 to 92\%). PCR-based methods to detect $O$. viverrini and $C$. sinensis eggs in stool have been developed, but are not commercially available. Other new methods for diagnosis based on the detection of parasite antigens in stool via dot-blot hybridization techniques are also being developed but are not widely 
available (Wongratanacheewin et al, 2001). Laboratory examination: Blood samples of patients' only with clonorchiasis were detected for circulating antigen $(\mathrm{CAg})$ with DotELISA, counted for esoinophilic granulocytes, and tested for alanine aminotransferase (ALT). Number of esoinophilic granulocytes above $0.5 \times 10^{9} / \mathrm{L}$ was regarded as increased, and the value of alanine aminotransferase (ALT) above 30u/L were judged as abnormal. Examination by B-mode ultrasonography abdominal part of patients only with $C$. sinensis eggs in their stools was examined (Wang et al, 2004).

Imaging: Ultrasound abnormalities may be detected among both symptomatic and asymptomatic individuals include gallbladder enlargement and/or sludge, bile duct inflammation and/or fibrosis, and hepatomegaly (Köster et al, 2016). Ultrasound may also detect aggregates of flukes as non-shadowing echogenic foci within bile ducts. In another study of 316 Korean patients with clonorchiasis, four sonographic findings distinguished patients with active disease from controls: increased periductal echogenicity, floating echogenic foci in the gallbladder, diffuse intrahepatic bile ducts dilatation, and gallbladder distention. Ultrasound abnormalities often normalize following treatment. In endemic areas, the finding of floating echogenic foci in the gallbladder one year after praziquantel treatment suggested re-active clonorchiasis. For evaluation of relapsing cholangitis, CT scanning has been reported to be more sensitive than ultrasound, since CT can detect the dilated and thickened small intrahepatic bile ducts associated with recurrent pyogenic cholangitis. MRI can also be a useful diagnostic tool (Behairy et al, 2009). In one study of 26 Korean patients with clonorchiasis, MRI demonstrated intrahepatic bile duct abnormalities in $89 \%$ of cases. The distribution of findings included: mild dilation of the intrahepatic bile duct (81\%), "Too many intrahepatic bile ducts" (62\%), wall enhancement and thickening $(81 \%)$, filling defects and ductal stricture in intrahepatic bile duct $(24 \& 12 \%$, respectively), and extrahepatic ductal dilation (19\%). Hepatobiliary malignancy was $(35 \%)$ in patients. Cholangiography detected slender filling defects in dilated intrahepatic bile ducts with wall irregularities and tortuosity. Changes in ultrasound and laboratory values before and after CCA diagnosis were reported in patients occupationally exposed to organic solvents (Kubo et al, 2016).

Generally, CT, MRI, and ultrasound guidance are useful tools to identify liver flukes (Mortelé et al, 2004; Wahib et al, 2005). Meanwhile, diagnosis of liver flukes should prompt evaluation of family members with stool microscopy. Individuals with evidence of infection should be treated to avoid the risk of future complications, even in the absence of symptoms. Several promising diagnostic biomarkers from serum or bile, such as the metalloproteinases or serotonin, were suggested (Wang et al, 2016).

Pathogenesis: Generally, parasites of the biliary tract are a common cause of biliary obstruction in tropical countries and can lead to such serious complications as cholangitis and cholangiocarcinoma (Feng and Cheng, 2017). Liver fluke infections cause serious medical and veterinary diseases. Clonorchiasis and opisthorchiasis (viverrini) are particularly dangerous. They can survive for several decades in humans causing chronic inflammation of the bile ducts, epithelial hyperplasia, periductal fibrosis and bile duct dilatation. In many infections these symptoms cause further complications such as stone formation, recurrent pyogenic cholangitis and cholangiocarcinoma (Lim, 2011).

Opisthorchiasis is particularly the leading cause of cholangiocarcinoma in Thailand and the Lao People's Democratic Republic (Sripa et al, 2011). Clonorchiasis and opisthorchiasis are classified as Group 1 human biological agents (carcinogens) by International Agency of Research on Cancer (Kim et al, 2016). Chaiyadet et al. (2015) demonstrated that $O$. viverrini secreted extracellular vesicles are released in the secreted pro- 
ducts of carcinogenic liver flukes, which then are internalized by the cholangiocytes, subsequently driving cell proliferation and IL-6 secretion and promoting an inflammatory but simultaneously modulatory environment. These processes ultimately facilitate CCA development. In $C$. sinensis differential diagnosis includes acute hepatitis, cholecystitis, choledocholithiasis, cholangiocarcinoma, primary sclerosing cholangitis, and other parasites as schistosomiasis, strongyloidiasis, and ascariasis (Tang et al, 2016).

Treatment: There were some controversies as to treatment. Praziquantel $(75 \mathrm{mg} / \mathrm{kg}$ ora1 ly in three divided doses for two days) gave cure rate $>90 \%$ (Jong et al, 1985). Side effects of treatment include headache, dizziness, insomnia, nausea, and vomiting (Avgustinovich et al, 2019). Lower dose regimens are better tolerated but, were less effective for the clonorchiasis treatment. Praziquantel $(40 \mathrm{mg} / \mathrm{kg}$ as a single dose) showed to be effective for opisthorchiasis treatment (cure 91 to 95\%), and has been used successfully in communities receiving mass treatment. Xiao et al. (2011) in experimentally infected rats confirmed that mebendazole given orally at a single dose of $150 \mathrm{mg} / \mathrm{kg}$ caused potential effect against juvenile (14day-old) and adult $C$. sinensis, without or less effect was obtained from albendazole under the same dose levels, but the extension course of treatment enhanced the albendazole effect. They added that a single effective dose of mebendazole and tribendimidine against $C$. sinensis in rats were similar with a broad window, while the praziquantel window was narrow. Albendazole $(10 \mathrm{mg} /$ $\mathrm{kg}$ orally for 7 days) was associated with the good efficacy for clonorchiasis ( $>90 \%$ cure) and the modest efficacy for opisthorchiasis (63\% cure rate), without severe adverse reactions during the treatment period (YingYan et al, 2018). Mebendazole (30mg/kg for 20 to 30 days) were associated with cure rates of 89 to $94 \%$ for opisthorchiasis. Clinical symptoms of the infection may take months to resolve, although typically eggs disappear from the stool within one week of treatment. $\mathrm{Xu}$ et al. (2011) in vitro reported that praziquantel and tribendimidine caused strong in-vitro killing effect on $C$. sinensis adults with minimal concentration of levamisole killed them 50 times higher than that of the tribendimidine. The higher concentrations of artemether and artesunate showed slower action to reduce the worm activity and killed part of them. The higher concentrations of albendazole and mebendazole exhibited no killing effect on $C$. sinensis, besides stimulate the motor activity of worm oral sucker.

Other interventions as the management of acute cholangitis by reliable diagnose as early as possible to initiate appropriate treatments to reduce mortality and morbidity (Lan Cheong Wah et al, 2017). Cholangitis may require antibiotics, biliary drainage, and/or surgery is a must. Burman et al. (2017) reported that cholestasis and disease progression can lead to cirrhosis; extrahepatic complications including dyslipidemia, metabolic bone disease, and fat-soluble vitamin deficiency. Ursodeoxycholic acid is a well-established therapy. Endoscopic therapy helped in the management of biliary complications caused by these parasites (Rana et al, 2007).

Endoscopic therapy has helped in management of biliary complications caused by these parasites (Akcakaya et al, 2006). Ascaris lumbricoides worms, which normally reside in the jejunum, are actively motile and can invade the papilla, thus migrating into the bile duct and causing biliary obstruction. Endoscopic retrograde cholangiopancreatography is a useful diagnostic tool with potential for therapeutic management of the biliary ascariasis. Clonorchis sinensis worms infection cause complications as intrahepatic stones, recurrent pyogenic cholangitis, cirrhosis, cholelithiasis, pancreatitis, as well as the cholangiocarcinoma (Valero et al, 2012). Opisthorchis viverrini, Opisthorchis felineus, and Dicrocoelium dendriticum are closely related to $C$. sinensis and cause cholangiocarcinoma (Pakharukova and Mordvinov, 2016). Zhuang et al. (2019) conclu- 
ded that although the risk of a second primary malignancy (SPM) in patients with CCA was significantly increased, the presence of SPM did not shorten overall survival and disease specific survival of patients with CCA, possibly due to the relatively poorer survival of patients with CCA.

Statistically, NCI (2013) in Thailand reported that cholangiocarcinoma (CCA) is a malignant neoplasm arising from the bile duct epithelia with up to 20,000 people died each year. It is one of the commonest cancers in Thailand, especially in north eastern part, where the prevalence of liver fluke infection was high. Age standardized incidence rate showed the highest incidence in Khon Kaen, Ghouri et al. (2015) in USA re-ported that the incidence and mortality of the CCA in North America had increased dramatically in the past decades. In USA, there were approximately 5,000 new cases diagnosed each year. Age-adjusted rates for CCA were highest among Hispanics \& Asians (2.8-3.3/ $100,000)$ \& lowest among non-Hispanic whites \& blacks $(2.1 / 100,000)$. Men showed a slightly greater mortality than women (1.9 \& 1.5/100,000, respectively). ICD-10 (2015) in England reported that initial report covering England only, showed CCA data for 4 years, 2010-2013, with incidence figures, 2010:1,832; 2011:1,864; 2012: 1,945; 2013: 1,965 \& mortality figures, 2010:1,720; 2011: 1,844; 2012:2,018; 2013: 2,161 prompting following comment in the executive summary of the report: "A number of cancer sites stand out as having comparatively high mortality rates considering incidence rates, biliary tract cancers 3.64 vs. $3.58 / 100,000$ population respectively"

WorldCCADay (2019) declared that there must be an international effort to raise moreneeded awareness of cholangiocarcinoma, a devastating cancer that occurs in bile ducts in or outside the liver. This poorly understood and under researched disease is increasing in incidence globally; they believed that collaboration is the way forward. Khan et al. (2019) stated that cholangiocarcinoma is a heterogeneous disease raised from the complex interaction between the host specific genetic background and multiple risk factors, exhibiting geographical variation, with much higher incidence in parts of the Eastern world compared to the West. They added that despite the advancements in the knowledge of CCA etiology, in Western Countries about $50 \%$ of cases are miss-diagnosed without any identifiable risk factor. It is conceivable that other still undefined etiologic factors are responsible for the CCA recent increase (especially iCCA) worldwide.

\section{Conclusion and Recommendations:}

Parasitosis remain as the persistent public health worldwide problems and significant groups for carcinogenesis Three helminthes, namely, Clonorchis sinensis and Opisthorchis viverrini (liver fluke) as well as Schistosoma haematobium (blood fluke), are classified as Group 1 carcinogens by the WHO's International Agency for Research on Cancer. Until now, the cellular and molecular mechanisms linking fluke infections to cancer formation have yet to be defined, although many studies have focused on these mechanisms in recent years, and numerous findings were made in various aspects of parasite-associated cancers. Herein, we only introduce the fluke-induced cholangiocarcinoma (CCA) and bladder carcinoma and mainly focus on key findings in the last 5 years. Both oncogenic liver flukes: clonorchis sinensis, and Opitherchus veverrini, $O$. felineus species are infective to humans by eating raw fresh water fish and crabs. They are endemic worldwide and affecting millions of humans in mostly asymptomatic form that requires treatment. There is strong evidence that Egypt and Middle East inhabits the first intermediate host like Buthynia and therefore the diseases are present here. Eggs in stools are confusing with each other and with intestinal nematodes. Therefore serology tests are advised to be more available since they are still limited in USA. More studies confirmed their presence as of extreme importance, sine those oncogenic liver flukes are definitely carcinogenic causing 
cholangiocarcimoma in about $10 \%$ of cases with a very poor prognosis as usually discovered in an advanced stage and related to chronic inflammation and irritation by the parasite in common bile duct. There are varies clinical presentations of Clonorochis that may be confusing with other diseases like gastritis, cholangitis, biliary mud and gall stone pancreatitis, that mandates the search for a more sensitive and specific test. Infection of those liver flukes can be prevented by cooking or freezing freshwater fish; strict hygiene measures also decrease transmission. Health education about cooking fish adequately, hygiene improvement to prevent water contamination, and stool examination followed by treatment of identified cases to eliminate human host reservoirs. Also, identifying animal reservoirs like cats, dogs and animals in slaughter houses helps to confirm its endemicity, localizing hosts and areas of affection for proper management. The FDA recommends the following for fish preparation or storage to kill any parasites: Cooking fish adequately (to an internal temperature of at least $145^{\circ} \mathrm{F}\left(63^{\circ} \mathrm{C}\right)$. Freezing fish by any of the following ways; $1-$ at $-4^{\circ} \mathrm{F}\left(-20^{\circ} \mathrm{C}\right)$ or below for at least 7 days (total time);or, 2at $-3^{\circ} \mathrm{F}\left(-35^{\circ} \mathrm{C}\right)$ or below until solid, and storing at $-31^{\circ} \mathrm{F}\left(-35^{\circ} \mathrm{C}\right)$ or below for at least 15hrs, or, 3 -at $-31^{\circ} \mathrm{F}\left(-35^{\circ} \mathrm{C}\right)$ or below until solid and storing at $-4^{\circ} \mathrm{F}\left(-20^{\circ} \mathrm{C}\right)$ or below for at least 24 hours.

Now what about Egypt and local countries?

\section{References}

Akcakaya, A, Sahin, M, Karakelleoglu, A, Okan, I, 2006: Endoscopic stenting for selected cases of biliary fistula after hepatic hydatid surgery. Surg. Endosc. 20, 9:1415-8.

Aksorn, N, Roytrakul, S, Kittisenachai, S, Leelawat, K, Chanvorachote, $P$, et al, 2018: Novel potential biomarkers for Opisthorchis viverrini infection and associated cholangiocarcinoma. In Vivo 32, 4:871-8

Al Karawi, MA, el Sheikh Mohamed, AR, al Shahri, MG, Yasawy, MI, 1993: Endoscopic sphincterotomy in acute gallstone pancreastitis and cholangitis: A Saudi Hospital experience. Hepatogastroenterol. 40, 4:396-401.

Al-Karawi, M, Qattan, N, 1992: Clonorchis si- nensis: A case report. Ann. Saudi Med. 12, 1:935.

Avgustinovich, D, Tsyganov, M, Vishnivetskaya, G, Kovner, A, Sorokina, I, 2019: Effects of supramolecular complexation of praziquantel with disodium glycyrrhizinate on the liver fluke Opisthorchis felineus: an in vitro and in vivo study. Acta Trop. Mar 11. pii: S0001-706X (18) 31428-1

Behairy, NH, Dorgham, MA, Khaled, SA, 2009: Accuracy of routine magnetic resonance imaging in meniscal and ligamentous injuries of the knee: comparison with arthroscopy. Int. Orthop. 33, 4: 961-7

Burman, BE, Jhaveri, MA, Kowdley, KV, 2017: An update on the treatment and follow-up of patients with primary biliary cholangitis. Clin. Liver Dis. 21, 4:709-23.

Chai, JY, Darwin Murrell, K, Lymbery, AJ, 2005: Fish-borne parasitic zoonoses: Status and issues. In.t J. Parasitol. 35, 11/12:1233-54.

Chaiyadet, S, Sotillo, J, Smout, M, Cantacessi, C, Jones, MK, et al, 2015: Carcinogenic liver fluke secretes extracellular vesicles that promote cholangiocytes to adopt a tumorigenic phenotype. J. Infect. Dis, 212, 10:1636-45.

Choi, MH, Ge, T, Yuan, S, et al, 2005: Correlation of egg counts of Clonorchis sinensis by three methods of fecal examination. Korean $\mathbf{J}$. Parasitol. 43:115-7.

Choi, MH, Ryu, JS, Lee, M, et al, 2003: Specific and common antigens of Clonorchis sinensis and Opisthorchis viverrini (Opisthorchidae, Trematoda). Korean J. Parasitol. 41:15-63.

Dao, HTT, Dermauw, V, Gabriël, S, Suwannatrai, A, Tesana, S, et al, 2017: Opisthorchis viverrini infection in the snail and fish intermediate hosts in Central Vietnam. Acta Trop. 170: 120-5.

Fattakhov, RG, Ushakov, AV, Stepanova, TF, Ianovich, VA, Kopylov, PV, 2012: Epizootiological characteristics of clonorchiasis foci in the Amur River ecosystem in the Jewish autonomic region. Meditsinskaia Parazitol. I Parazit. Bolezni 4:15-8.

Feng, M, Cheng, X, 2017: Parasite-Associated Cancers (Blood Flukes/Liver Flukes). Adv. Exp. Med. Biol. 1018:193-205.

Geller, SA, 2014: Autoimmune hepatitis: Histopathology. Clin. Liver Dis. 3, 2:19-23.

Ghouri, YA, Mian, I, Blechacz, B, 2015: Cancer review: Cholangiocarcinoma. J. Carcinog. 14:1-3163.151940. eCollection 2015. 
Harinasuta, C, Harinasuta, T, 1984: Opisthorchis viverrini: Life cycle, intermediate hosts, transmission to man and geographical distribution in Thailand. Arzneimittelforschung 34, 9B:1164 $-7$.

Hung, NM, Dung, DT, Anh, NT, Van, PT, Thanh, BN, 2015: Current status of fish-borne zoonotic trematode infections in Gia-Vien District, Ninh Binh Province, Vietnam. Parasit. Vetors 8, 1:216-9.

ICD-10, 2015: International Statistical Classification of Diseases and Related Health Problems $10^{\text {th }}$ Revision coding created by the WHO http:// apps.who.int/classifications/icd10/browse/en\#/C 22.1

Jong, EC, Wasserheit, JN, Johnson, RJ, et al, 1985: Praziquantel for the treatment of Clonorchis/Opisthorchis infections: Report of a doubleblind, placebo-controlled trial. J. Infect. Dis.152: 63-7.

Kaewkes, S, Elkins, DB, Sithithaworn, P, Haswell-Elkins, MR, 1991: Comparative studies on the morphology of the eggs of Opisthorchis viverrini and lecithodendriid trematodes. Southeast Asian J. Trop. Med. Publ. Hlth. 22:623-30.

Keiser, J, Utzinger, J, 2005: Emerging foodborne trematodiasis. Emerg. Infect. Dis. 11: 1507-12.

Khan, SA, Tavolari, S, Brandi, G, 2019: Cholangiocarcinoma: Epidemiology and risk factors. Liver Int. Mar 9. doi: 10.1111/liv.14095

Kim, TS, Pak, JH, Kim, JB, Bahk, YY, 2016: Clonorchis sinensis, an oriental liver flu$\mathrm{ke}$, as a human biological agent of cholangiocarcinoma: A brief review. BMB Reports 49, 11: 590-7.

Köster, L, Shell, L, Illanes, O, Lathroum, C, Neuville, K, et al, 2016: Percutaneous ultrasound-guided cholecystocentesis \& bile analysis for the detection of Platynosomum spp. induced cholangitis in cats. J. Vet. Int. Med. 30, 3:787-93

Kubo, S, Takemura, S, Tanaka, S, et al, 2016: Screening and surveillance for occupational cholangiocarcinoma in workers exposed to organic solvents. Surg. Today. 46:705-12.

Lan Cheong Wah, D, Christophi, C, Muralidharan, V, 2017: Acute cholangitis: Current concepts. ANZ J. Surg. 87, 7/8:554-9.

Lawley, TJ, Bielory, L, Gascon, P, et al, 1984: A prospective clinical and immunologic analysis of patients with serum sickness. N. Engl. J. Med. 311:1407-11.

Lim, JH, 2011: Liver flukes: The malady negle- cted. Korean J. Radiol. 12, 3:269-79.

Mairiang, E, Laha, T, Bethony, JM, et al, 2012: Ultrasonography assessment of hepatobiliary abnormalities in 3359 subjects with Opisthorchis viverrini infection in endemic areas of Thailand. Parasitol. Inter. 61:208-11.

Morsy, ATA, Al-Mathal EM, 2011: Clonorchis sinensis a new report in Egyptian employees returning back from Saudi Arabia. J. Egypt. Soc. Parasitol. 41:221-6.

Morsy, TA, EI Maridi, NA, 1978: Incidence of parasitic infection in Baqaa, Jordan. J. Egypt. Soc. Parasitol. 8, 2:347-51.

Mortelé, KJ, Segatto, E, Ros, PR, 2004: The infected liver: radiologic-pathologic correlation. Radiographics 24, 4:937-55.

NCI, 2013: The National Cancer Institute: Currently available national statistics for CCA in Thailand. This data is from a national census performed in 2008.

Nguyen, HM, Tatonova, YV, Madsen, H, 2018: Infections by hepatic trematodes in cats from slaughterhouses in Vietnam. J. Parasitol. 104, 3:306-9.

Pakharukova, MY, Mordvinov, VA, 2016: The liver fluke Opisthorchis felineus: biology, epidemiology and carcinogenic potential. Trans. R. Soc. Trop. Med. Hyg. 110, 1:28-36

Pinto, HA, Gonçalves, NQ, López-Hernandez, D, Murillo, EA, Alan L. Melo, AL, 2018: The life cycle of a zoonotic parasite reassessed: Experimental infection of Melanoides tuberculata (Mollusca; Thiaridae) with Centrocestus formosanus (Trematoda: Heterphyidae). PLoS One 13, 4:e0194161.

Pozio, E, Armignacco, O, Ferri, F, Gomez Morales, MA, 2013: Opisthorchis felineus, an emerging infection in Italy and its implication for the European Union. Acta Trop. 126, 1:5462.

Qian, MB, Utzinger, J, Keiser, J, Zhou, XN, 2016: Clonorchiasis. Lancet 387:800.

Ran, X, Yin, B, Ma, B, 2017: Four major factors contributing to the intrahepatic stones. Gastroenterol. Res. Pract. Article ID 7213043; 5pages. https://doi.org/10.1155/7213043

Rana, SS, Bhasin, DK, Nanda, M, Singh, K, 2007: Parasitic infestations of the biliary tract. Curr. Gastroenterol. Rep. 9, 2:156-64.

Rim, HJ, 2005: Clonorchiasis: An update. J. Helminthol. 79:269-72.

Saijuntha, W, Sithithaworn, P, Kaitsopit, N, Andrews, R, Petney, T, 2014: Liver flukes: $\mathrm{Cl}$ - 
onorchis and Opisthorchis. In: Digenetic Trematodes. Toledo, R, Fried, B, New York (USA): Springer, ISBN: 978-1-49-390914-8.

Sirisinha, S, Sahassananda, D, Bunnag, D, Rim, HJ, 1990: Immunological analysis of Opisthorchis and Clonorchis antigens. J. Helminthol. 64, 2:133-8.

Sithithaworn, P, Haswell-Elkins, MR, Mairiang, $P$, et al, 1994: Parasite-associated morbidity: Liver fluke infection and bile duct cancer in northeast Thailand. Int. J. Parasitol. 24:833-8.

Sripa, B, Bethony, JM, Sithithaworn, P, Kaewkes, S, Mairiang, E, et al, 2011: Opisthorchiasis and Opisthorchis-associated cholangiocarcinoma in Thailand and Laos. Acta Trop. 120, 1:S158-68.

Stauffer, WM, Sellman, JS, Walker, PF, 2004: Biliary liver flukes (Opisthorchiasis and Clonorchiasis) in immigrants in the United States: Often subtle and diagnosed years after arrival. J. Travel Med. 11, 3:157-9.

Tan, WB, Shelat, VG, Diddapur, RK, 2009: Oriental liver fluke infestation presenting more than 50 years after immigration. Ann. Acad. Med. Singapore 38:735-9.

Tang, ZL, Huang, Y, Xin-Bing, Y, 2016: Current status and perspectives of Clonorchis sinensis and clonorchiasis: Epidemiology, pathogenesis, economics, prevention and control. Infect. Dis. Povert. 5, 1: 71-8.

Tangkawattana, S, Tangkawattana, P. 2018: Reservoir animals and their roles in transmission of Opisthorchis viverrini. Adv. Parasitol. 101:69 -95 .

Tohamy, AA, Mohamed, SM, 2006: Chromosomal studies on two Egyptian freshwater snails, Cleopatra and Bithynia (Mollusca-Prosobranchiata). Arab J. Biotech. 9, 1:17-26.

Tsui, WMS, Chan, YK, Wong, CT, Lo, YF, Yeung, YW, et al, 2011: Hepatolithiasis and the syndrome of recurrent pyogenic cholangitis: clinical, radiologic, and pathologic features. Seminars in Liver Disease 31, 1:33-48.

Upatham, ES, Viyanant, V, Kurathong, S, et al, 1984: Relationship between prevalence and intensity of Opisthorchis viverrini infection, and clinical symptoms and signs in a rural community in north-east Thailand. Bull. WHO 62:451-6.

Valero, V, Cosgrove, D, Herman, JM, Pawlik, TM, 2012: Management of perihilar cholangiocarcinoma in the era of multimodal therapy. Expert. Rev. Gastroenterol. Hepatol. 6, 4:481-95.
Wahib, AA, Seif El Nasr, MS, Mangoud, AM, El Shazly, AM, Morsy, ATA, 2005: The liver function profile in PCR-RNA Egyptian HCVpatients and normal controls. J. Egypt. Soc. Parasitol. 35, 2:451-66.

Wang, KX, Zhang, RB, Cui, YB, et al, 2004: Clinical and epidemiological features of patients with clonorchiasis. World J. Gastroenterol. 10: 446-50.

Wang, B, Chen, L, Chang, HT, 2016: Potential diagnostic and prognostic biomarkers for cholangiocarcinoma in serum \& bile. Biomark. Med. 10:613-9.

Whitton, B, 1982: Rivers, Lakes and Marshes. By Hodder \& Staughton, London.

WHO, 1995: Control of Foodborne Trematode Infections: Report of a study group. Tech. Rep. Ser. 849:1-8.

Wongratanacheewin, S, Pumidonming, W, Sermswan, RW, Maleewong, W, 2001: Development of a PCR-based method for the detection of Opisthorchis viverrini in experimentally infected hamsters. Parasitology 122:175-80

WorldCCADay, 2019: World Cholangiocarcinoma Day@WorldCCADay

Xiao, SH, Xue, J, Xu, LL, Zhang, YN, Qiang, HQ, 2011: Comparative effect of mebendazole, albendazole, tribendimidine, and praziquantel in treatment of rats infected with Clonorchis sinensis. Parasitol Res. 108, 3:723-30.

Xu, LL, Xue, J, Zhang, YN, Qiang, HQ, Xiao, SH, 2011: In vitro effect of seven anthelmintic agents against adult Clonorchis sinensis. Zhongguo Ji Sheng Chong Xue Yu Ji Sheng Chong Bing Za Zhi 29, 1:10-5.

Ying-Yan, Z, Ting-Jun, X, Man, W, Yue-Yi, F, Le, L, 2018: Prevalence of Clonorchis sinensis infection and effect of albendazole treatment among residents in two communities of Zhongshan City. Zhongguo Xue Xi Chong Bing Fang Zhi Za Zhi. 30, 2:219-21.

Yossepowitch, O, Gotesman, T, Assous, M, et al, 2004: Opisthorchiasis from imported raw fish. Emerg. Infect. Dis. 10:21-2.

Zheng, S, Zhu, Y, Zhao, Z, Wu, Z, Okanurak, K, et al, 2017: Liver fluke infection and cholangio-carcinoma: A review. Parasitol. Res. 116, 1: 11-19.

Zhuang, L, Yan, X, Meng, Z, 2019: Second primary malignancy in patients with cholangiocarcinoma: A population-based study. Cancer Manag. Res. 11:1969-83. 